\title{
Heat shock gene resides in a pore neighborhood
}

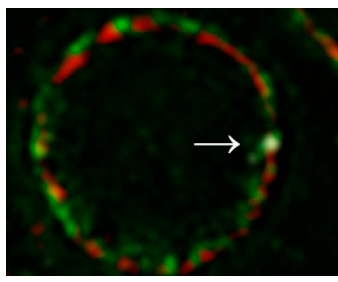

Super-resolution microscopy shows the hsp-16.2 promoter (white) colocalizing with pores (green) at the nuclear periphery (red).

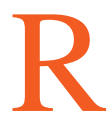

ohner et al. describe how the heat shock gene $h s p-16.2$ associates with nuclear pore complexes in C. elegans cells.

Genes can occupy specific positions within the nucleus, and their localization may change upon activation or repression. Developmentally regulated genes in C. elegans, for example, move from the periphery to the interior of nuclei in response to gene induction. In contrast, Rohner et al. found that the worm stress response gene $h s p$-16.2 tended to localize near the nuclear envelope in its inactive state and became even more enriched at the nuclear periphery when induced by heat shock.

Transgenes containing the minimal $h s p-16.2$ promoter showed similar localization patterns, indicating that the promoter region, Breaking down Arp2/3 function
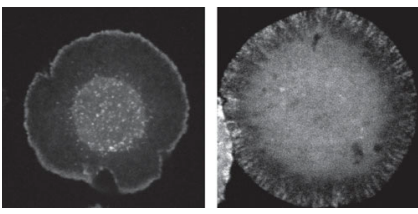

Compared with wild-type Arp3 (left), a hydrolysis-defective Arp3 mutant (right) remains associated with an actin network that extends further inwards from the edge of a spreading S2 cell.

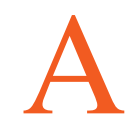

TP hydrolysis by the Arp2/3 complex promotes the disassembly of lamellipodial actin networks, Ingerman et al. reveal.

The Arp2/3 complex generates branched actin networks and lamellipodial cell protrusions by nucleating new actin filaments from the sides of preexisting ones. The complex contains two actin-related proteins, Arp2 and Arp3, that bind ATP in a conserved nucleotide-binding pocket, like actin itself. ATP hydrolysis by actin destabilizes polymerized actin filaments, but the effect of ATP hydrolysis by Arp2 and Arp3 remains unclear.

Ingerman et al. made hydrolysis-defective versions of Arp2

\section{PEX26 takes the direct route}
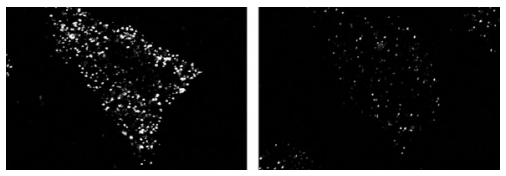

Compared with a semi-intact control cell (left), the delivery of PEX26 (white) to peroxisomes is reduced in PEX3's absence (right).

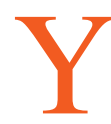

agita et al. describe how an integral membrane protein is delivered directly to mammalian peroxisomes instead of passing through the ER.

Peroxisomal membrane proteins can take either a direct or indirect path to their organelle, and both routes are controlled by mammalian cytosolic chaperone PEX19 and its peroxisomal receptor PEX3, or their homologues. The budding yeast protein Pex15, for example, follows an indirect route. Its C-terminal transmembrane domain is first inserted into the ER by an ATPase called Get3, and the protein is then transported to peroxisomes by yeast Pex19 and Pex3. In mammals, PEX19 is also required to deliver PEX26, the orthologue of Pex15, to rather than any other sequences or the gene's chromosomal location, controls $h s p$-16.2 positioning. Using super-resolution microscopy and chromatin immunoprecipitation, the researchers found that the promoter localized near nuclear pore complexes before gene induction and then increased its association with the pores upon heat shock.

hsp-16.2's association with nuclear pores was mediated by several factors that bind to the gene's promoter. Knocking down heat shock transcription factor 1 or expressing heat-sensitive mutants of RNA polymerase II abolished the gene's peripheral localization. $h s p-16.2$ positioning also required the polymeraseassociated protein ENY-2, which binds to nuclear pores and promotes mRNA processing and export. $h s p$-16.2's peripheral localization may therefore speed up heat shock protein production in times of stress. But senior author Susan Gasser thinks that, in the uninduced state, pore-associated factors might help limit gene expression by promoting mRNA turnover.

Rohner, S., et al. 2013. J. Cell Biol. http://dx.doi.org/10.1083/jcb.201207024.

and Arp3. These mutants were still able to nucleate actin filaments and support the assembly of branched actin networks in cells. But hydrolysis-defective Arp2/3 complexes took longer to dissociate from actin filaments, thereby delaying — but not preventing - network turnover. Complexes containing mutant versions of both Arp2 and Arp3 were even slower to initiate network disassembly than complexes containing just one hydrolysis-defective subunit, indicating that Arp2 and Arp3 have independent roles in network turnover.

Hydrolysis-deficient Arp2/3 complexes were also able to assemble branched actin networks in vitro, and these networks were more resistant to disassembly by the actin-severing protein cofilin. The researchers now want to investigate how cofilin and related proteins contribute to the disassembly of lamellipodial actin networks and to determine how their activities are coordinated with ATP hydrolysis by actin and the Arp2/3 complex.

Ingerman, E., et al. 2013. J. Cell Biol. http://dx.doi.org/10.1083/icb.201211069.

peroxisomes. But whether PEX26 makes a stopover in the ER was unknown.

Yagita et al. found that PEX19 binds to PEX26 in the cytosol and delivers the protein to peroxisomes by docking onto PEX3. Unlike in yeast, PEX26 didn't accumulate in the ER of mammalian cells lacking PEX19. Moreover, PEX26 showed no interaction with TRC40, the mammalian homologue of Get3, and still localized to peroxisomes when this ATPase was inhibited.

Mammalian PEX26 therefore takes a different, more direct path to peroxisomes than its budding yeast counterpart. This route is determined by PEX26's relatively hydrophilic transmembrane domain, which avoids capture by TRC40, and by a stretch of basic residues in PEX26's C terminus that interact with PEX19. Senior author Yukio Fujiki now wants to investigate how PEX26 is integrated into the peroxisomal membrane after PEX19 and PEX3 have recruited it to the organelle's surface.

Yagita, Y., et al. 2013. J. Cell Biol. http://dx.doi.org/10.1083/jcb.201211077. 